OTU-26 DATA DRIVEN SERVICE EVALUATION OF AN IBD HELPLINE

${ }^{1}$ Louise Downey*, ${ }^{1}$ Richard Harris, ${ }^{1}$ Martin McDonnell, 'Anne Sanderson, 'Marion Bettey, ${ }^{1}$ Maria Rubio-Padilla, ${ }^{2}$ Florina Borca, ${ }^{2}$ Hang Phan, ${ }^{1}$ Richard Felwick, ${ }^{1}$ Fraser Cummings, ${ }^{1}$ Markus Gwiggner. 'Gastroenterology Department, University Hospital Southampton, UK; ${ }^{2}$ NIHR Biomedical Research Centre, University Hospital Southampton, UK

\subsection{6/gutjnl-2019-BSGAbstracts. 121}

Introduction We present a data driven service evaluation of the use of an Inflammatory Bowel Disease (IBD) telephone helpline service at a single UK IBD centre (UHS). We demonstrate how such evaluation can highlight service improvement needs to maximise efficiency and improve patient care.

Methods IBD patients at UHS can call our helpline and leave a message for one of our IBD specialist nurses to respond to the following working day. All helpline activity is electronically logged in the electronic health record. Anonymised data was systematically extracted for the first 6 months of 2016, 2017 and 2018. Calls were categorised as follows: 1) flare call symptoms relating to IBD, 2) follow up call - following change in medication/results, 3) medication enquiries, 4) administrative calls and 5) unsuccessful unanswered calls.

Results A total of 7,046 calls were analysed. Activity increased through the study period with 1627, 2676 and 2743 attempted/successful outgoing calls made to 598, 785 and 882 unique patients in the first six months of 2016, 2017, 2018 respectively (table 1 ).

Abstract OTU-26 Table 1 Breakdown of call numbers and time taken for first 6 months of each year

\begin{tabular}{llll}
\hline \multicolumn{4}{l}{ Number of calls (Time in hours) } \\
\hline Flare & 2016 & 2017 & 2018 \\
Follow-up & $602(210.5)$ & $912(324.2)$ & $967(352.1)$ \\
Medication & $282(90.6)$ & $457(123.1)$ & $450(124.8)$ \\
Administration & $223(52.4)$ & $305(79.9)$ & $385(112.3)$ \\
Unsuccessful & $104(19.4)$ & $181(38.3)$ & $172(42.8)$ \\
Total & $416(19.7)$ & $821(53.1)$ & $769(56.1)$ \\
& 1627 & 2676 & 2743 \\
& $(392.6)$ & $(618.6)$ & $(688.1)$ \\
\hline
\end{tabular}

In total $28.5 \%$ of calls were unsucessful. The 416,821 and 769 unsuccessful calls were made to 228,388 and 384 unique patients respectively with associated preparation time of 19.7 , 53.1 and 56.1 hours (hrs). Call success was similar for each weekday but showed a trend to improvement towards the end of each day (after 4pm).

Administrative calls for 2016, 2017 and 2018 included patients requesting test results which generated 52 (10.5 hrs), $87(20.0 \mathrm{hrs})$ and 105 (26.5 hrs) calls and appointment queries which generated 23 (4.1 hrs), 41 (7.9 hrs) and 21 (5.4 hrs) calls respectively.

Conclusions Use of our IBD helpline has increased over time. When using incoming call requests as a marker of activity it is important to recognise that approximately half of these require follow-up calls to review results and treatment plans. This must be considered when designing services. Time wasted in unsuccessful calls is substantial and novel methods to reduce this are needed. Online access to results and appointments may reduce total calls. An online portal (My Medical Record) for medication information, results and admin queries as well as a pre-call text message are being introduced at UHS.

\section{OWE-01 THE EFFECT OF INFLIXIMAB DOSE ESCALATION IN INFLAMMATORY BOWEL DISEASE PATIENTS WITH ANTIBODIES TO INFLIXIMAB}

Gloria SZ Tun*, Laura Marshall, Laura Thompson, Rebecca Campbell, Melissa F Hale, Thean S Chew, Alenka J Brooks, Kerry Robinson, Alan J Lobo. Sheffield Teaching Hospitals NHS Foundation Trust, Sheffield, UK

\subsection{6/gutjnl-2019-BSGAbstracts. 122}

Introduction Infliximab (IFX) dose escalation (DE) can be used in patients with inflammatory bowel disease with loss of response (LOR) or subtherapeutic drug levels. However, the long-term benefit of DE remains unclear, especially in those with antibodies to infliximab (ATI).

Aim To assess the effect of DE in patients with ATI on drug level, clinical response and ATI status.

Methods IFX and ATI trough levels (Immundiagnostik, UK) were measured at each IFX infusion in patients from May 2016 at a large referral centre and results retrospectively reviewed in December 2018. DE comprised a reduction in dose interval between maintenance infusions $<8$ weeks \pm an increase dose of IFX to $10 \mathrm{mg} / \mathrm{kg}$. Clinical remission was defined for Crohn's disease (CD) as Harvey Bradshaw Index $\leq 4+$ C-reactive protein $\leq 5 \mathrm{mg} / \mathrm{L}$ and for ulcerative colitis as Simple Clinical Activity Index $\leq 4$. ATI $>10 \mathrm{mg} / \mathrm{L}$ is considered 'positive' by manufacturer. Positive ATI that resolved within two consecutive infusions were defined as transient.

Results 78 patients were dose escalated (41 male; 40 CD; age 17-81; 51 on immunosuppression): 48 for LOR and 30 to optimise therapeutic drug monitoring levels. 73 received DE for a median 36 weeks (range 4-140). 5 patients stopped IFX after 1 further dose: 2 for LOR and 3 for infusion reaction (IR). At the time of DE, 31/78 (40\%) patients had ATI >10 $\mathrm{mg} / \mathrm{L}$ (ATI+). In patients with ATI $\leq />10 \mathrm{mg} / \mathrm{L}$, DE significantly increased drug levels: median IFX levels of 1.3 and 0.9 respectively at baseline to 3.1 and 3.5 at week 24 (figure 1). After DE, 13/33 ATI+ had a fall in ATI $\leq 10 \mathrm{mg} / \mathrm{L}$ : median pre-DE ATI $23 \mathrm{mg} / \mathrm{L}$ (range 10-86), median post-DE ATI 9

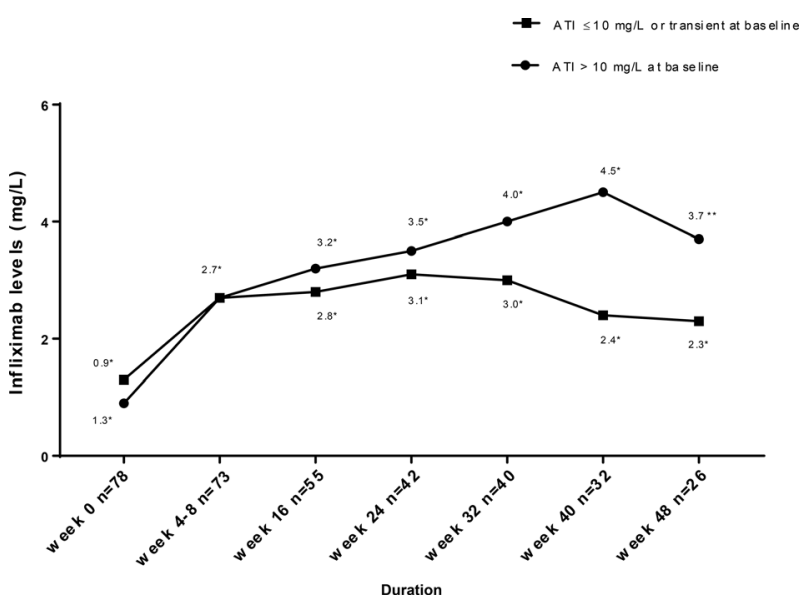

Abstract OWE-01 Figure 1 Median IFX levels following dose escalation

IFX levels at follow up compared to baseline ${ }^{*} p<0.01{ }^{* *} p=0.06$ 